\title{
16-Bit Microcomputers The advantages of 16-bit microcomputers
}

\author{
KENNETH E. HANSEN \\ Psych Systems, 206 Medical Tower, Norfolk, Virginia 23507
}

\begin{abstract}
Microcomputers have great applicability for psychological laboratories. While it is usual to limit discussions of microcomputers to 8-bit equipment, 16-bit machines have several advantages. In many situations, these systems prove to be more efficient and cost effective.
\end{abstract}

In recent years, large-scale integration (LSI) technology has made a significant impact on the computing industry. LSI components are used to construct microprocessors that can be produced in large quantities at a very low price. Microprocessors are currently being used in a wide variety of applications such as peripheral controllers, intelligent terminals process control systems, communications equipment, and graphics systems because of their low cost and great versatility. Probably the most popular application of a microprocessor is the stand-alone computing system.

There are numerous types of microprocessors available, each with its own specific characteristics, but they can be grouped into two broad categories. The first consists of systems based on a single-chip microprocessor (Pavel, 1978). The majority of microcomputer systems in the hobby market place fall in this category. The second grouping encompasses systems based on microprocessors that are comprised of multiple LSI chips. These systems, which usually have a 16-bit word size, are more common in the professional computing industry.

Microcomputers of both types have many advantages. However, most of the relevant literature has focussed on the 8-bit equipment. In this paper, the case for 16-bit processors is presented.

\section{8-BIT MICROPROCESSORS}

Most of the single-chip microprocessors have an 8-bit word size (Osborne, 1976). Some of the primary manufacturing sources include Intel (8080A, 8048), Motorola (MC6800), Commodore (MCS6502), Zilog (Z80, Z80A), National Semiconductor (SC/MP), and Fairchild (F80) (Osborne, 1978). Numerous publications are available that describe the various characteristics of these systems. ${ }^{1,2,3,4}$ Eight-bit microcomputers are well suited for many laboratory applications, including instrument control (Anders, Anné, \& Sanders, 1977; Durrett, 1978; Murray \& Lawler, 1978).

Because of the low cost of this type of equipment, ${ }^{5}$ it can often be used in network configuration approaches (Polson, 1978).

\section{Disadvantages}

Despite the popularity of 8-bit processors, they have several disadvantages. Some of the companies producing them are underfinanced, and consequently, they may market components that are not thoroughly tested and are sometimes unreliable. Many vendors have adopted the $\mathrm{S}-100$ bus as a standard for components. The S-100, however, is not a standard bus. Signals used by some components are not compatible with others. For example, a very fast processor would be constrained to operate at slower speeds to retain compatibility with the other modules. Much of the available hardware performs appropriately, but there are potential problems.

It is often stated that the limiting factor in the use of 8-bit systems rests with the software. This is only partially true. Some applications do not require sophisticated software. For example, scientific instrumentation often needs only a small number of assembly language instructions for appropriate control. However, assembly language is not desirable for many applications because of the great detail involved. Higher level languages generally allow applications programs to be developed more rapidly and with greater ease.

The most popular higher level languages for microcomputers are a set of languages derived from BASIC. ${ }^{6}$ Despite its popularity, BASIC has many disadvantages. Most forms of BASIC utilize an interpreter that requires a substantial amount of memory overhead. Furthermore, structured programs are difficult to write and variable names are extremely restricted. Since no overall standard has been adopted for BASIC, many varieties exist that have unique features. ${ }^{7,8,9}$ In many situations, FORTRAN may be a more appropriate language. However, FORTRAN is not available on many systems.

\section{6-BIT MICROPROCESSORS}

Sixteen-bit microprocessors are frequently developed to emulate a minicomputer line (e.g., DEC LSI-11, Data General Micro-NOVA). These systems offer the power of a minicomputer at a substantially lower price. They are often overlooked as being expensive alternatives to 8-bit processors, in spite of the fact that in many cases 16-bit systems may prove to be less expensive. They are more aligned with the professional computing community, and continued product support is available from the vendor. OEM distributors and 
second-source vendors have caused their marketing to become very competitive. Therefore, it is possible to build a 16-bit system at a substantial savings by selecting components from various manufacturers.

The Digital Equipment Corporation (DEC) LSI-11 is the prime example. MDB Systems ${ }^{10}$ produces excellent interface modules for the LSI-11. These interfaces feature DIP switch-selectable parameters, eliminating the need to resolder jumpers for configuration changes. MDB also second sources the processor, backplane (bus), and most other hardware components. It also specializes in producing special user-designed interfaces. All MDB products come with a 1-year warranty, quick delivery, and a significant discount in price. Both Intel ${ }^{11}$ and Monolithic Systems ${ }^{12}$ produce memory modules for the LSI-11. These components are advantageous when maximum memory ( $56 \mathrm{~K}$ bytes) is required,,$^{3}$ or when many devices are to be installed on the bus. A $48 \mathrm{~K}$-byte module requires only a double slot and one bus load. Furthermore, these modules have DIP switches to select $4 \mathrm{~K}$ blocks of memory. If a chip becomes faulty, the memory board can be reconfigured at $4 \mathrm{~K}$ less memory to operate until the chip is replaced. Data Systems Design ${ }^{14}$ manufactures a floppy-disk system utilizing Shugart drives. The controller card for this disk system also contains a built-in bootstrap and bus termination that eliminates the need for the REV-11 card and reduces the need for one dual slot on the bus. Data Systems has also announced a magnetic bubble storage system that emulates the floppy-disk system. All of these components are totally compatible with DEC hardware and transparent to its software.

Standard operating system software for the LSI-11 includes the RT- $11^{15}$ monitor and associated utility routines (editor, assembler, linker, file-utility programs, library control program, on-line debugger, and batch processor). RT-11 is a single-user operating system designed for interactive programs and on-line applications. This monitor can operate either in a single-job or foreground-background environment. An extensive macro library is supplied that helps to simplify the task of assembly language programming. Higher level languages supported include BASIC, multiuser BASIC (up to eight users), and FORTRAN. A laboratory package providing FORTRAN subroutines to control A/D-D/A signals, real-time clock, and Schmitt triggers is also supported. All of the software is very well documented.

Alternative system software is also available. An LSI-11 system can be downloaded from a larger minicomputer in the PDP-11 family and operate under a powerful multitasking operating system, RSX-11S. ${ }^{16}$ LSX (Lycklama, 1977), a subset of UNIX (Thompson \& Ritchie, 1974), is a single-user operating system available from Bell Laboratories. LSX, like UNIX, is written in the structured, higher level language C. Some of the advantages of using LSX are that applications programs can be written in $\mathrm{C}$, the command interpreter from UNIX is supported, and a hierarchical file system is available, thus making an LSX system ideal for control of dedicated experiments. $\mathrm{TSX}^{17}$ is a timesharing executive that operates under RT- 11 . This software system incorporates a swapping-type algorithm that allows users to utilize all available memory. User programs are swapped in and out of memory depending on program status. The system supports up to 10 users. These alternate operating systems extend the environments and applications for an LSI-11 system.

Sixteen-bit microcomputer systems that are second sourced by many other vendors can often be assembled at prices that are comparable to 8-bit microprocessorbased systems. For example, CRDS ${ }^{18}$ offers an LSI-11 system with dual Shugart drives and is functionally identical to the PDP-11V03. The system has a unit price of $\$ 4,290$. A similar configuration with an 8-bit processor costs about the same. Yet, this 16-bit machine offers a large and powerful instruction set, sophisticated diagnostic software, versatile (and debugged) operating system software and utility routines, extensive documentation, support for several higher level languages, and ongoing overall product support. Sixteen-bit microcomputers are often excellent bargains that should not be overlooked.

\section{SELECTION FACTORS}

The criteria for selecting a microcomputer are similar to those for larger systems. However, there is currently a tendency to select a microcomputer system based solely on initial purchase price without consideration of system requirements. In many applications, the hardware components constitute as little as $20 \%$ of the total cost involved. Therefore, an inadequate system may prove to be very expensive in the long run.

It is important to carefully evaluate the primary application for which the computer system is being chosen and to work from a functional application description. The application description normally provides initial system specifications. For example, specifications for a simple data acquisition system may include sampling frequency, number of samples, and period over which sampling is to take place. These factors, in turn, determine required computer characteristics such as cycle time, memory size, and mass storage. A detailed analysis of the application generally shortens the amount of time necessary for appropriate system selection.

When defining the application, it is necessary to consider all related available resources, for these are varied and often overlooked when making selection decisions. For example, software that is available free of charge from various groups and individuals might be a factor for determining which languages are to be 
supported on the equipment to be selected. In-house maintenance resources and adequate diagnostic software can be important in determining whether to purchase equipment from a manufacturer that does not provide extensive product support, but usually offers equipment at a lower price. Conversely, it may be advantageous to purchase equipment from a vendor that offers an extensive maintenance service rather than developing an in-house maintenance program. An evaluation should be made of the current software development expertise. It may prove efficient to purchase developed software packages rather than to perform the development in-house. Such considerations are often very important in choosing equipment.

\section{DISCUSSION}

LSI technology has made available some invaluable tools for a wide variety of laboratory applications. The uses of microcomputers will undoubtedly increase as additional features become available and systems become more powerful. The increasing amount of interest directed toward the microcomputer industry will stimulate new product development and maintain the competitiveness of the market place. These are all very favorable factors for the consumer.

The hobby computer industry has made an important impact on computing by helping to remove the mystique surrounding computers, raising the level of understanding and knowledge about computers, and establishing an important market place. Often the components utilized in a hobby computer system can find direct and important applications in the laboratory.

However, selecting a hobby computer is fundamentally different from selecting a professional computer. Hobby computers are most often selected on the basis of the initial investment, while performance and reliability are generally secondary factors. Applications may only be vaguely defined. Often an assumption is made in personal computing that developing applications is an entertaining and educational experience, so software development is not considered a cost. These considerations do not hold true when selecting a computer for a professional application.

Economic factors are very important when selecting a computer system. But there are certain aspects of computer uses that are difficult to attach a dollar figure to. For example, a system that costs $\$ 2,000$ and takes 1 man-year of programming to become operational is more expensive than a $\$ 10,000$ system that is running the same task in a week. How much is actually saved on an inexpensive system if the documentation is inadequate? Can a programmer be more productive if writing programs in a higher level language such as FORTRAN than in assembly language? Such considerations do not pertain to all applications. They merely illustrate that many factors besides hardware costs play an important role in selection. Considering the many factors involved in selection of a microcomputer system, 16-bit machines are less expensive and provide greater performance in many situations.

\section{REFERENCES}

Anders, R. J., AnNÉ, A., \& SANders, D. B. A microcomputer system for on-line acquisition of neuromuscular data. Proceedings of the First Annual Symposium on Computer Application in Medical Care. New York: Institute of Electrical and Electronic Engineers, Inc, 1977.

DURRETT, H. J. Inexpensive microcomputer systems for research and instruction: A dream or reality? Behavior Research Methods \& Instrumentation, 1978, 10, 345-351.

LYCKLAma, H. UNIX on a microprocessor. AFIPS Conference Proceedings 1977 National Computer Conference, 1977, 46, 237-242.

MurRay, D. M., \& LAWLER, J. E. A KIM-I mictoprocessor interface for aversive conditioning applications using multiple subjects. Behavior Research Methods \& Instrumentation, 1978, 10, 334-339.

OsBorne, A. An introduction to microcomputers: Some real products (Vol. 2). Berkley: Osborne, 1976.

OsBorne, A. Who's who in personal computing-A partial guide to microcomputer hardware and vendors. Mini-Micro Systems, May 1978. Pp.70-73.

PAvel, M. What is a microprocessor? Behavior Research Methods \& Instrumentation, 1978, 10, 238-240.

Polson, G. Microprocessors: Their impact on real-time computing in psychology. Behavior Research Methods \& Instrumentation, 1978, 10, 139-147.

Thompson, K., \& Ritchie, D. M. The UNIX Time-Sharing System. Communications $A C M, 17$, July 1974. P. 365.

\section{NOTES}

1. Popular Electronics, 1 Park Avenue, New York, New York 10017. 03458.

2. Byte, 70 Min Street, Petersborough, New Hampshire

3. Kilobaud, Petersborough, New Hampshire 03458.

4. Personal Computing, Benwill Publishing Corporation, 167 Corey Road, Brookline, Massachusetts 02146.

5. Psych Systems, Suite 206, Medical Tower, Norfolk, Virginia 23507.

6. BASIC is a registered trademark of the Trustees of Dartmouth College.

7. BASIC-11 Language Reference Manual, Digital Equipment Corporation, Maynard, Massachusetts.

8. Microterm-II Extended Disk Basic Programming Manual, Digi-Log Systems, Inc., Horsham, Pennsylvania.

9. Altair BASIC Reference Manual, MITS, 1975.

10. MDB Systems, Inc., 1995 N. Batavia Street, Orange, California 92665.

11. Intel Corporation, Memory Systems Division, $1302 \mathrm{~N}$. Mathilda Avenue, Sunnyvale, California 94086.

12. Monolithic Systems Corporation, 14 Inverness Drive East, Englewood, Colorado 80110.

13. The KD11-F processor board contains the LSI-11 microprocessor and $8 \mathrm{~K}$ by tes of memory, thus an additional $48 \mathrm{~K}$ by tes of memory is necessary for a maximum memory configuration. With the LSI-11/2 now becoming available, it is possible to save one more dual slot in the backplane, since all $56 \mathrm{~K}$ bytes of memory is available on a single dual-slot board and the LSI-11/2 requires only a dual slot. 
14. Data Systems Design, Inc., 3130 Coronado Drive, Santa Clara, California 95051.

15. DEC has recently announced an enhanced version of RT-11, RT-11 Version 3. The software includes new hardware support and software features. The monitor will support APL, BASIC, DIBOL, FOCAL, and FORTRAN IV.

16. RSX-11S is a multitasking operating system for dedicated systems. RSX-11S must be developed on a larger PDP-11 minicomputer operating under either RSX-11M or RSX-11D.
RSX-11 supports many higher level languages, including BASIC and FORTRAN.

17. S \& H Computer Leasing, Inc., 3709 Trimble Road, Nashville, Tennessee 37215.

18. Charles River Data Systems, Inc., 4 Tech Circle, Natick, Massachusetts 01760 .

(Received for publication June 28, 1978.) 